\title{
DCIS does not need treatment... really?
}

\author{
Kevin S. Hughes ${ }^{1,2}$
}

Received: 10 October 2015 / Accepted: 12 October 2015/Published online: 17 October 2015

(C) Springer Science+Business Media New York 2015

A recent article on ductal carcinoma in situ (DCIS) by Dr. Steven Narod, one of the associate editors of this journal, has generated tremendous publicity and controversy. Dr. Narod used the Surveillance Epidemiology, and End Results (SEER) database to examine 10- and 20-year breast cancer-specific mortality in patients following a diagnosis of DCIS [1]. Not surprisingly, he found that by the 20-year benchmark, the overall risk of breast cancer-specific mortality was $3.3 \%$ (956 patients). What was surprising, however, was that he also found that 517 of these patients did not experience an intervening invasive episode in the ipsilateral or contralateral breast prior to death. This finding was unexpected because the prevailing wisdom is that DCIS does not metastasize without first recurring as an invasive cancer. He also found that the type of treatment (mastectomy vs. excision, with or without adjuvant therapy) had no discernible impact on mortality. Unfortunately, Dr. Narod's study was misconstrued by the media, and potentially others, to mean that surgery is no longer recommended for early-stage breast cancer, as evidenced in the New York Times report entitled "Doubt Is Raised Over Value of Surgery for Breast Lesion at Earliest Stage" [2]. Actually, nothing could be further from the truth. Dr. Narod's quote in the Times, "I think the best way to treat D.C.I.S. is to do nothing," followed the proviso, "After a surgeon has removed the aberrant cells." In fact, Dr. Narod

Kevin S. Hughes

kshughes@partners.org

1 Harvard Medical School, Avon Comprehensive Breast Evaluation Center, Massachusetts General Hospital, 55 Fruit Street, Yawkey 7, Boston, MA 02114, USA

2 Bermuda Cancer Genetics and Risk Assessment Clinic, Hamilton, Bermuda was not recommending for the DCIS to be left in situ. He was suggesting the DCIS be removed, but without further treatment after surgery.

The controversy surrounding Dr. Narod's article stems from confusion about three tenets of breast cancer treatment: (1) Surgery is necessary to treat breast cancer; (2) Mortality is not the only consideration or rationale for determining the course or endpoint of treatment; and (3) DCIS is not a single disease.

Surgery is necessary to treat breast cancer All curable breast cancer patients require surgery, with a subset needing additional adjuvant therapies (i.e., hormonal therapy, chemotherapy, radiation therapy, or biologics). While I agree with the Scottish surgeon, John Hunter (1728-1793), who said that surgery "is like an armed savage who attempts to get that by force which a civilized man would get by stratagem," [3] this does not change the fact that all breast cancer patients will die of their disease without surgical intervention. Savage or not, surgery is the sine qua non and our most effective stratagem in the cure of breast cancer. Nevertheless, recognizing the attendant morbidity of surgical resection, surgeons have spent the last several decades trying to decrease morbidity without decreasing the rate of cure. Hence, the rising interest in breast conservation, sentinel node biopsy, breast reconstruction, nipple-sparing mastectomy, and so on. While none of these advances has increased survival beyond the cure rates Halstead achieved with radical mastectomy, they have all reduced trauma to the patient without compromising cure rates. The real issue, and point of Dr. Narod's study, is not whether surgery is needed, but how much surgery is needed, and how much adjuvant therapy is necessary after surgery.

A recent paper by Sagara et al. which relied on the same SEER database used by Dr. Narod, also addressed the role of 
surgery in DCIS [4]. Sagara reported that women with intermediate and high-grade DCIS who did not undergo surgery had a higher breast cancer-specific mortality than those treated with surgery, whereas no mortality advantage was found for surgically removing low-grade DCIS. What are we to conclude from this information? Considering that all of these patients listed as not having surgery actually did have some invasive procedure (core biopsy or excisional biopsy) where some or all of the DCIS was removed, the conclusion perhaps should have been that minimal excision of DCIS is not sufficient to prevent mortality for intermediate and high-grade DCIS, while minimal or incomplete excision of low-grade DCIS may be sufficient to prevent death. The authors made no mention of in-breast recurrence in their report.

Mortality is not the only consideration or rationale for determining the course or endpoint of treatment Long-term survival is not the only measure of success in breast cancer treatment. Quality of life is also of tremendous importance. We need to weigh the negative impact on quality of life associated with large excisions, radiation, and poor cosmetic outcome against the likelihood of an in-breast recurrence. The type of in-breast recurrence, invasive or DCIS, and the manner by which the in-breast recurrence will be treated (mastectomy vs. conservation) are other important factors. A recurrence of DCIS that can be treated conservatively has far less negative impact on quality of life than an invasive recurrence necessitating a mastectomy. In determining what is an acceptable rate of in-breast recurrence, we might look to the European Society of Breast Cancer Specialists (EUSOMA) guidelines [5], which suggest that an acceptable rate of in-breast recurrence is $1-2 \%$ per year, or we could look at the rate of contralateral breast cancer as a possible benchmark. McCormick et al. [6] found that lowto-intermediate-grade DCIS treated with tamoxifen but without radiation had a 7-year in-breast recurrence risk of $6.7 \%$ (95\% confidence interval 3.4-10), not dissimilar to the contralateral breast cancer incidence of $4.8 \%(95 \%$ confidence interval 2.0-7.7) and well below the 1-2\% per annum suggested by EUSOMA. If a local therapy (surgery plus/minus tamoxifen) produces a rate of in-breast recurrence that is less than 1-2\% per year, and if that rate is similar to the baseline rate of breast cancer occurrence in the untreated breast, is that not sufficient evidence to recommend that approach?

DCIS is a spectrum of disease Perhaps the most important issue is that DCIS is not a single disease. To suggest that DCIS does not need to be treated is as misleading as saying that all DCIS must be treated with mastectomy. We need to be cognizant of the specific type of DCIS, and the characteristics of the specific patient, to determine the best treatment. As to type, Sagara's article implies that high and intermediate-grade DCIS require extensive surgery, but low-grade DCIS might not. As to patient characteristics, Narod's article suggests that African Americans and young women with DCIS should be treated more aggressively.

The debate is not about the need for surgery, but rather about how much tissue should be removed during surgery, and whether additional therapies, such as hormonal therapy or radiation, should be added to surgery. SEER reviews are, to a certain extent, an example of "Big Data," a rather blunt instrument that provides general results, rather than "Personalized Medicine" a highly nuanced precision approach to care. Both approaches have value and limitations.

In the age of personalized medicine, treatments should be tailored to the specific type of DCIS and to the specific characteristics of the patient. Numerous factors must be considered in determining the best treatment plan. Surgical management is categorized as biopsy alone, lumpectomy with minimal margins, lumpectomy with wide margins, and mastectomy. Adjuvants to surgery include none, radiation therapy, hormonal therapy, and combined radiation and hormonal therapy. DCIS can be classified grossly (age and race of the patient), more specifically (grade, the presence or absence of necrosis, estrogen receptor status and size), and most specifically (genomic characteristics of the DCIS). All of these factors should be analyzed against expected outcomes to determine the optimal approach to treatment.

\section{Should a mastectomy ever be done for DCIS?}

For the person who has never faced a newly diagnosed patient with DCIS, it is easy to reject radical surgery. We know that lumpectomy and mastectomy have essentially the same survival for breast cancer, but we forget the allimportant proviso, "in patients for whom lumpectomy is possible." Lumpectomy is not always possible. Patients eligible for lumpectomy are those who can have their cancer excised with acceptable surgical margins, leaving behind a cosmetically acceptable breast [7]. For a woman with extensive calcifications that take up a substantial portion of the breast, however, lumpectomy is not possible. Doing nothing is not an option because unless the lesion is excised, there can be no reasonable assurance that the pathology is $100 \%$ DCIS. A core biopsy showing DCIS may represent sampling error, and the lesion may actually contain areas of invasive cancer [8]. Excision is required to confirm the diagnosis. Since the excision will not produce a cosmetically acceptable result, under these circumstances mastectomy with reconstruction is the better option. 


\section{If lumpectomy is chosen, how extensive should the surgery be?}

Lumpectomy margins have been a point of controversy for decades. When I first started out in surgical oncology, Dr. Fisher's landmark study [9] on breast conservation had just been published. Based on this, I was content with margins of "no ink on tumor" for the rest of the 1980s and early 1990s, but owing to varying opinions of the community at large [10] and local custom, I did $1 \mathrm{~mm}$ in the late 1990s and $2 \mathrm{~mm}$ in the early 2000s. In 2014, the consensus guidelines on resection margins for invasive cancer were published in the Journal of Clinical Oncology [11]. The consensus stated that "no ink on tumor" was sufficient for invasive cancer, which is once again my standard practice. I eagerly await the consensus for DCIS margins, which I am hopeful will also say "no ink on tumor."

It is important to remember that once your margin is negative, the size of any additional margin has no impact on survival. The maximum margin of normal breast tissue you can obtain is with mastectomy, and yet, mastectomy has no survival advantage over lumpectomy with a minimal margin. Margin size has minimal impact on in-breast recurrence in invasive cancer where radiation is used [12]. However, for DCIS, especially if not treated with radiation, it is still believed that the greater the margin, the lower the incidence of in-breast recurrence [13]. Thus, for DCIS, margin has two conflicting roles: (1) the greater the margin the less the in-breast recurrence and (2) the greater the margin, the worse the cosmetic outcome. Lumpectomy is a cosmetic/quality of life operation. We do not recommend lumpectomy because it cures more cancer; it does not. We recommend it because it provides a better quality of life. If QOL is our goal, then the fewer the re-operations, the fewer the mastectomies, and the better the cosmetic outcome, the more useful lumpectomy will be. Minimal margins fulfill these criteria with minimal tissue removed, minimal reoperations for close margins, and minimal immediate mastectomies performed for "close" margins.

On the downside, smaller margins in DCIS, particularly if not treated with radiation, will likely lead to slightly higher rates of in-breast recurrence, adversely affecting QOL. However, the effect on QOL is most severe when it results in mastectomy, but may have less impact if the outcome is a delayed lumpectomy and radiation many years after diagnosis. If the initial treatment is lumpectomy without radiation, one can usually do lumpectomy with radiation sometime in the future if an in-breast recurrence happens.

\section{Should radiation ever be used in addition to surgery?}

The addition of radiation to lumpectomy has no real impact on survival unless the rate of invasive in-breast recurrence exceeds $10 \%$ at 5 years [14], which is essentially never going to occur with DCIS. Initially, radiation increases the cost of treatment and adds morbidity. Delayed morbidity in terms of telangiectasia, decreased breast size, and discomfort appears over time. This financial and personal cost must be weighed against the decrease in in-breast recurrence that can be achieved with radiation. While shorter and/or lower dose radiation [15-17] will decrease cost and may decrease morbidity and inconvenience, their use still must be weighed against the expected benefit.

The rate of in-breast recurrence is sufficiently elevated in high-grade DCIS to justify radiation after lumpectomy, and it is sufficiently low in low-grade DCIS that radiation may possibly be safely avoided. It is less clear how to use radiation after lumpectomy in intermediate-grade DCIS. Genomic analysis has helped more objectively estimate the risk of in-breast recurrence with DCIS and may be useful in determining the need for radiation [18].

\section{Should hormonal therapy ever be used as an adjuvant to surgery?}

Hormonal therapy (tamoxifen, aromatase inhibitors) has the benefit of both decreasing the risk of in-breast recurrence and decreasing the risk of contralateral breast cancer. These drugs also have costs and associated side effects. While hormonal therapy has never been shown to decrease the death rate from DCIS, its rather mild side effect profile and its benefits in terms of preventing recurrent or new primary breast cancer make it a reasonable approach to consider in patients undergoing lumpectomy. Once again, one needs to consider the use of hormonal therapy in conjunction with other factors. It should only be used in estrogen receptor (ER)-positive breast cancer [19], and it may be more beneficial in patients who have not received radiation [20].

\section{Can surgery be avoided all together?}

Few people are suggesting that DCIS should not be excised. Even those suggesting such an approach are doing so as part of a clinical trial for highly selected cases. There are precious little data regarding women who have not had an excision for the reason that an invasive procedure is needed to diagnose DCIS. Hence, no cohort exists that has 
not had some or all of the disease removed to make the diagnosis.

Proponents of no treatment point to Page's study published in 1982 as proof that most DCIS does not progress to invasive cancer [21]. However, what that paper actually said was that $28 \%$ of DCIS treated by biopsy only will develop invasive cancer. Once again, limited surgery is not the absence of surgery. In addition, as discussed earlier, Sagara [4] has shown that the absence of surgery will lead to unnecessary deaths in those with intermediate and highgrade DCIS. Before embarking on a course of no surgery, one should consider the risks against the potential gain. Excising a limited DCIS with a minimal margin is a small operation with minimal cosmetic effects. This surgery can markedly decrease the rate of local recrudescence. In the situation where there are extensive calcifications and a core showing DCIS, excision will have cosmetic consequences, but without excising, the tumor you will not be able to confirm that $100 \%$ of the calcifications represent DCIS. A non-surgical approach runs the risk of leaving invasive cancer untreated [22].

DCIS is a spectrum of disease with varying malignant potentials, which requires varying approaches and treatments. Mastectomy remains a very appropriate treatment for extensive disease that cannot be removed without causing a major cosmetic deficit. Lumpectomy plus radiation is appropriate for those with high-grade DCIS or a high Oncotype score. Lumpectomy without radiation may be very appropriate for a low-grade DCIS or a DCIS with a low Oncotype score. For these patients, a larger margin and/or the use of hormonal therapy may be worthwhile. The use of excision with positive margins or biopsy only with no further therapy should be considered experimental, and an ongoing study may eventually answer questions regarding the safety of that approach. In short, we must consider the biology of the DCIS, and the life expectancy and co-morbidities of the patient in order to help the patient to choose the right management.

\section{References}

1. Narod SA, Iqbal J, Giannakeas V, Sopik V, Sun P (2015) Breast cancer mortality after a diagnosis of ductal carcinoma in situ. JAMA Oncol 1(7):888-896. doi:10.1001/jamaoncol.2015.2510

2. Doubt is raised over value of surgery for breast lesion at earliest stage, New York Times (2015) http://www.nytimes.com/2015/ 08/21/health/breast-cancer-ductal-carcinoma-insitu-study.html?_ $\mathrm{r}=1$. Accessed 29 Sept 2015

3. McDonald P (ed) (2004) Oxford dictionary of medical quotations, 1st edn. Oxford University Press, Oxford

4. Sagara Y, Mallory MA, Wong S et al (2015) Survival benefit of breast surgery for low-grade ductal carcinoma in situ: a population-based cohort study. JAMA Surg 150:739-745
5. Rutgers EJ (2001) Quality control in the locoregional treatment of breast cancer. Eur J Cancer 37:447-453

6. McCormick B, Winter K, Hudis C et al (2015) RTOG 9804: a prospective randomized trial for good-risk ductal carcinoma in situ comparing radiotherapy with observation. J Clin Oncol 33:709-715

7. Schwartz GF, Veronesi U, Clough KB, et al. (2006) Proceedings of the consensus conference on breast conservation, April 28 to May 1, 2005, Milan. Cancer 107:242-250

8. Lee JW, Han W, Ko E et al (2008) Sonographic lesion size of ductal carcinoma in situ as a preoperative predictor for the presence of an invasive focus. J Surg Oncol 98:15-20

9. Fisher B, Redmond C, Fisher ER et al (1985) Ten-year results of a randomized clinical trial comparing radical mastectomy and total mastectomy with or without radiation. $\mathrm{N}$ Engl $\mathrm{J}$ Med 312:674-681

10. Taghian A, Mohiuddin M, Jagsi R, Goldberg S, Ceilley E, Powell S (2005) Current perceptions regarding surgical margin status after breast-conserving therapy: results of a survey. Ann Surg 241:629-639

11. Moran MS, Schnitt SJ, Giuliano AE et al (2014) Society of surgical oncology-American Society for Radiation Oncology consensus guideline on margins for breast-conserving surgery with whole-breast irradiation in stages I and II invasive breast cancer. J Clin Oncol 32:1507-1515

12. Moran MS, Schnitt SJ, Giuliano AE et al (2014) Society of surgical oncology-American Society for Radiation Oncology consensus guideline on margins for breast-conserving surgery with whole-breast irradiation in stages I and II invasive breast cancer. Int J Radiat Oncol Biol Phys 88:553-564

13. Silverstein MJ, Lagios MD (2010) Choosing treatment for patients with ductal carcinoma in situ: fine tuning the University of Southern California/Van Nuys prognostic index. J Natl Cancer Inst Monogr 2010:193-196

14. Clarke M, Collins R, Darby S et al (2005) Effects of radiotherapy and of differences in the extent of surgery for early breast cancer on local recurrence and 15-year survival: an overview of the randomised trials. Lancet 366:2087-2106

15. Hathout L, Hijal T, Theberge V et al (2013) Hypofractionated radiation therapy for breast ductal carcinoma in situ. Int $\mathrm{J}$ Radiat Oncol Biol Phys 87:1058-1063

16. Taghian AG, Kozak KR, Doppke KP et al (2006) Initial dosimetric experience using simple three-dimensional conformal external-beam accelerated partial-breast irradiation. Int J Radiat Oncol Biol Phys 64:1092-1099

17. Vaidya JS, Wenz F, Bulsara M et al (2014) Risk-adapted targeted intraoperative radiotherapy versus whole-breast radiotherapy for breast cancer: 5-year results for local control and overall survival from the TARGIT-A randomised trial. Lancet 383:603-613

18. Solin LJ, Gray R, Baehner FL et al (2013) A multigene expression assay to predict local recurrence risk for ductal carcinoma in situ of the breast. J Natl Cancer Inst 105:701-710

19. Allred DC, Anderson SJ, Paik S et al (2012) Adjuvant tamoxifen reduces subsequent breast cancer in women with estrogen receptor-positive ductal carcinoma in situ: a study based on NSABP protocol B-24. J Clin Oncol 30:1268-1273

20. Rudloff U, Jacks LM, Goldberg JI et al (2010) Nomogram for predicting the risk of local recurrence after breast-conserving surgery for ductal carcinoma in situ. J Clin Oncol 28:3762-3769

21. Page DL, Dupont WD, Rogers LW, Landenberger M (1982) Intraductal carcinoma of the breast: follow-up after biopsy only. Cancer 49:751-758

22. Kurniawan ED, Rose A, Mou A et al (2010) Risk factors for invasive breast cancer when core needle biopsy shows ductal carcinoma in situ. Arch Surg 145:1098-1104 